\title{
Use of Non-ophthalmic Ultrasound for Evaluation of Retinal Detachment in Patients with Opaque Media
}

\author{
Pipat Kongsap, ${ }^{1}$ Nuanrat Kongsap ${ }^{2}$ \\ ${ }^{1}$ Department of Ophthalmology, and ${ }^{2}$ Department of Radiology, Prapokklao Hospital, \\ Chanthaburi, Thailand
}

\begin{abstract}
Aim: To examine the accuracy of non-ophthalmic ultrasonography for detecting retinal detachment in eyes with opaque media

Methods: This was a prospective observational study conducted over a 1-year period. Eyes with opaque media in the form of a mature cataract, occluded pupils, or vitreous haemorrhage that precluded visualisation of the fundus were examined using non-ophthalmic ultrasound to detect retinal detachment. The clinical findings reported by the ophthalmologist were compared with the radiologist's assessment.

Results: Of the 95 eyes of 94 patients enrolled in the study, 86 had mature cataract, 7 had vitreous opacity, and 2 had eye trauma. Fifteen eyes (15.8\%) showed evidence of posterior segment pathology on ultrasonography, with underlying conditions of retinal detachment $(n=7 ; 7.4 \%)$, posterior vitreous detachment $(n=5 ; 5.3 \%)$, asteroid hyalosis $(n=1 ; 1.1 \%)$, a vitreous traction membrane attached to the optic disc $(n=1 ; 1.1 \%)$, and intraocular foreign body $(n=1 ; 1.1 \%)$. Using non-ophthalmic ultrasonography, retinal detachment was detected in all 7 eyes, to achieve a sensitivity of $100 \%$ (95\% confidence interval, $56-100 \%$ ) and a specificity of 99\% (95\% confidence interval, 92-99\%).

Conclusions: Non-ophthalmic ultrasound is highly accurate for excluding retinal detachment, and may have a role in diagnosing retinal detachment in patients presenting with opaque media.
\end{abstract}

Key words: Eye, Retinal detachment, Ultrasonography

Asian J Ophthalmol. 2011;12:208-10.

\section{Introduction}

Ophthalmologists commonly use ultrasonography for evaluation of eyes, particularly when slit-lamp examination and fundoscopy are expected to provide insufficient data. ${ }^{1,2}$ The role of ultrasound in the detection of retinal detachment in eyes with opaque media has already been established. ${ }^{3,4}$ Generally, ophthalmologists use a $10-\mathrm{MHz}$ B-mode probe and an 8- to $10-\mathrm{MHz}$ A-mode probe to detect intraocular pathology. $A$ detached retina appears as a thin continuous acoustically opaque line of echoes, separate from and anterior to the echoes from the wall of the globe but connected to those from the optic nerve (Figures 1 and 2). However, this ophthalmic ultrasound equipment may not be available in the ophthalmology departments of some general hospitals that possess only conventional grey scale ultrasound equipment in the radiology departments.

Correspondence: Dr Pipat Kongsap, Department of Ophthalmology, Prapokklao Hospital, Chanthaburi 22000, Thailand. Tel: (66 3) 932 4975;

E-mail: pkongsap@yahoo.com or pkongsap@gmail.com
Radiologists use a 7.5- to $15-\mathrm{MHz}$ probe to detect systemic pathologies in the abdomen, chest, head, and neck. Although modern non-ophthalmic ultrasound equipment capable of producing better quality images has been developed, the sensitivity and specificity of modern radiological ultrasound equipment used for detection of ocular pathology have not been reported to date. The aim of this study was to evaluate whether the use of nonophthalmic ultrasound can accurately evaluate retinal detachment in eyes with opaque media.

\section{Methods}

A prospective observational study was performed on a convenience sample of patients, who presented between August 2009 and September 2010 to Prapokklao Hospital, Chanthaburi, Thailand. The inclusion criteria were presence of a mature cataract, vitreous haemorrhage, and/or corneal scar that obscured the visualisation of the retina by fundoscopy. The study was approved by the hospital institutional review board. Written informed consent was obtained from each patient. 
Figure 1. A funnel-shaped retinal detachment attached to the firm anchoring points of the ora serrata anteriorly and the optic nerve head posteriorly.

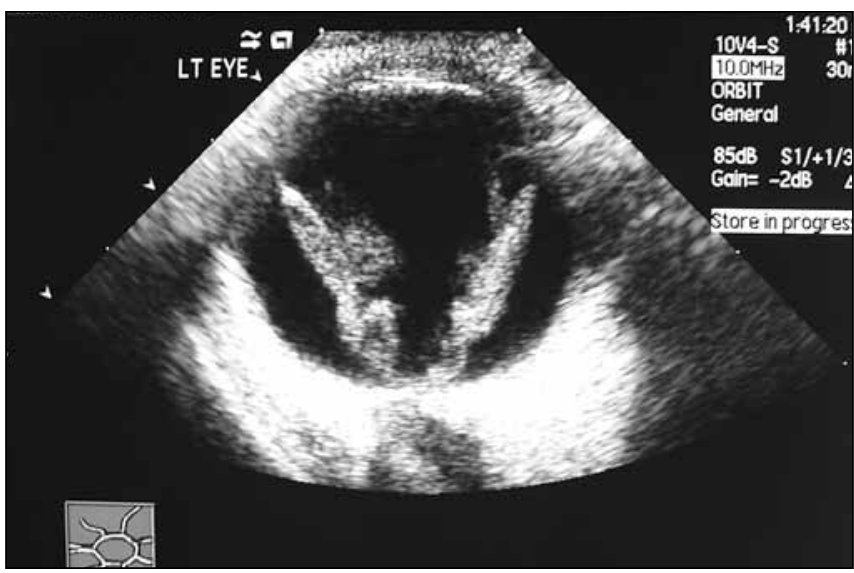

Ocular ultrasonographic examinations were performed by a radiologist using an ACUSON Sequoia 512 ultrasound system (Siemens, Munich, Germany) equipped with a 4- to 10-MHz linear probe (10V4 transducer) with the contact method. The probe was placed over the closed eyelid after applying a coupling gel. A radiologist who had a special interest in ocular ultrasonography was instructed to submit a data collection sheet that included printed results of the ultrasound examination and assessment of cases of retinal detachment prior to evaluation by an ophthalmologist. The clinical findings reported by the ophthalmologist were compared with the radiologist's assessment.

The ophthalmology assessment included visual acuity measurement and indirect fundoscopy examination with dilated pupils after cataract surgery, vitrectomy, or spontaneous resorption of vitreous haemorrhage. Only patients who were available for ophthalmological follow-up were included in the study. The sensitivity and specificity of non-ophthalmic ultrasonography was estimated by using $95 \%$ exact confidence intervals $(\mathrm{Cl})$. A false-positive result was defined as a positive ultrasound scan in the absence of clinical findings. $A$ false-negative result was defined as a negative ultrasound scan in the presence of clinical findings.

\section{Results}

Ultrasonography was performed for 95 eyes of 94 patients over 14 months. Among these patients, 86 had mature cataract, 7 had vitreous opacity, and 2 had eye trauma. Follow-up records were maintained for all 94 patients. Bilateral mature cataract in 1 patient necessitated the examination of both eyes. The patients' demographic data are shown in Table 1.

Fifteen eyes (15.8\%) showed evidence of some ultrasonographic posterior segment pathology. Retinal detachment (7 eyes; $7.4 \%$ ) was the most frequent abnormality detected; other abnormal findings were posterior vitreous detachment $(n=5 ; 5.2 \%)$, asteroid
Figure 2. A newly developed retinal detachment appearing as a taut linear opacity within the vitreous cavity that moved freely on a kinetic scan.

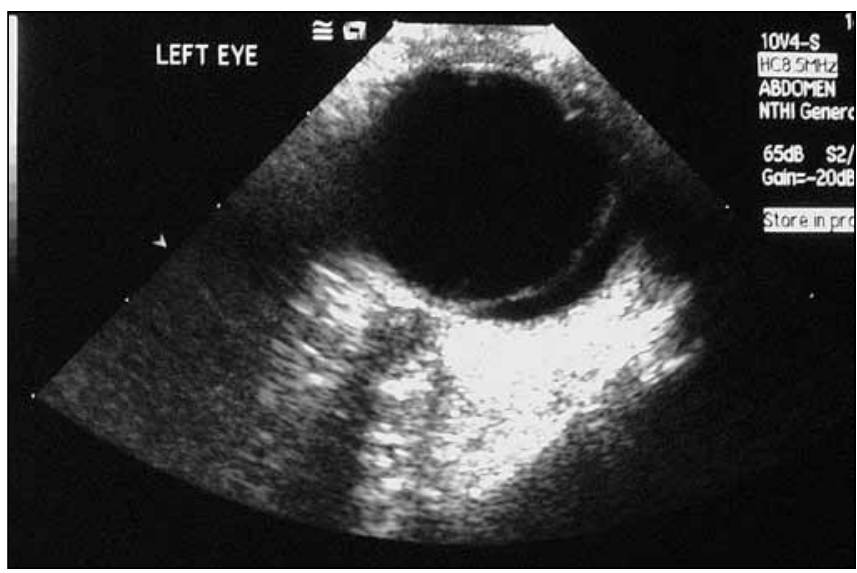

Table 1. Patients' demographic and clinical characteristics.

\begin{tabular}{|lc|}
\hline Characteristic & Number (\%) \\
\hline Number of patients & 94 \\
Number of eyes* & 95 \\
Mean age (SD) [years] & $63.98(9.37)$ \\
$\quad$ Range & $37-93$ \\
Sex & \\
Male & $45(47.4)$ \\
Female & $50(52.6)$ \\
Indication for ultrasound & \\
Lens opacity & $86(90.5)$ \\
Vitreous haemorrhage & $7(7.4)$ \\
Trauma & $2(2.1)$ \\
\hline
\end{tabular}

* One patient had bilateral mature cataract.

hyalosis ( $\mathrm{n}=1 ; 1.1 \%$ ), a vitreous traction membrane attached to the optic disc, which mimicked retinal detachment on ultrasound ( $n=1 ; 1.1 \%)$, and intraocular foreign body $(n=1 ; 1.1 \%)$.

The fundoscopy findings were in agreement with the confirmatory studies in all 7 eyes with ultrasonographic findings of retinal detachment. Thus, non-ophthalmic ultrasound had a sensitivity of $100 \%(95 \% \mathrm{Cl}, 56-100 \%)$. Of the 88 eyes that were judged to be free from retinal detachment by the ophthalmologist, 87 were judged identically by the radiologist for a specificity of $99 \%$ (95\% Cl, 92-99\%) [Table 2]. The positive predictive value was $88 \%$ $(95 \% \mathrm{Cl}, 47-99 \%)$ and the negative predictive value was $100 \%$ (95\% Cl, 95-100\%).

The only patient for whom there was disagreement about the diagnosis had diabetic vitreous haemorrhage with a vitreous traction membrane attached to the optic disc.

\section{Discussion}

Ophthalmic ultrasound can be potentially useful for diagnosing or excluding ocular pathologies such as globe rupture, lens dislocation, retrobulbar haemorrhage, intraocular foreign bodies, 
Table 2. Ultrasound and clinical findings for retinal detachment $(n=95)$.

\begin{tabular}{|lccc|}
\hline \multirow{2}{*}{ Ultrasound findings } & \multicolumn{3}{c|}{ Clinical findings } \\
\cline { 2 - 4 } & RD-positive & RD-negative & Total \\
\hline RD-positive & 7 & 1 & 8 \\
RD-negative & 0 & 87 & 87 \\
Total & 7 & 88 & 95 \\
& Number (\%) & $\mathbf{9 5 \%}$ confidence \\
& & interval \\
Sensitivity & $7 / 7(100)$ & $56-100$ \\
Specificity & $87 / 88(99)$ & $92-99$ \\
Positive predictive value & $7 / 8(88)$ & $47-99$ \\
Negative predictive value & $87 / 87(100)$ & $95-100$ \\
\hline
\end{tabular}

Abbreviation: $\mathrm{RD}=$ retinal detachment.

central retinal artery occlusion, retinal detachment, vitreous haemorrhage, and vitreous detachment. ${ }^{5-7}$ Ultrasound can also be used to evaluate chronic ocular pathologies such as tumour, anterior chamber disease, and retinal disease..$^{8-11}$ The ability of ocular ultrasound to assess the eye and its surrounding tissues in a rapid, accurate, safe, and non-invasive manner makes it a popular diagnostic tool. Interestingly, ocular ultrasound is usually not performed by radiologists, but by ophthalmologists or specialized technologists in ophthalmology clinics. However, this ophthalmic ultrasound equipment may not be available in all ophthalmology departments, and access may only be available for the conventional ultrasonographic equipment in the radiology department.

The single-purpose ophthalmic ultrasound machine and the 10-MHz probe system differ from the conventional non-ophthalmic ultrasonographic equipment. The 10V4 transducer used in paediatric and neonatal imaging facilitates high-frequency imaging that, in turn, provides better image quality. The frequency can be adjusted from 4 to $10 \mathrm{MHz}$. The $15 \mathrm{~L} 8$ transducer $(8-15 \mathrm{MHz})$ or the $8 \mathrm{~L} 5$ transducer $(5-8 \mathrm{MHz})$ can also be used for the evaluation of retinal detachment.

In this study, the non-ophthalmic ultrasound equipment available in the radiology department was used for the evaluation of retinal detachment before cataract or vitreoretinal surgery. In developing countries, ophthalmologists see many cases of mature or hypermature cataract or ocular injury for which fundoscopy is not useful because of the presence of an opaque media. Retinal and vitreous examinations may help ophthalmologists predict the postoperative visual prognosis and plan further management. In this study, the detection of retinal detachment with radiologic ultrasound was similar to that with ophthalmic ultrasound (sensitivity, 97.0100\%; specificity, 92.0-97.2\%). ${ }^{5,7}$ Considering the high specificity $(99 \%)$, and negative predictive value $(100 \%)$ of non-ophthalmic ultrasonography observed in this study, it is possible that nonophthalmic ultrasound can potentially be used for excluding retinal detachment in an eye with opaque media. These findings show that non-ophthalmic ultrasound, like ophthalmic ultrasound, can detect posterior vitreous detachment, asteroid hyalosis, and intraocular foreign bodies. ${ }^{6}$

Only 1 false-positive case was found - diabetic vitreous haemorrhage with a vitreous traction membrane attached to the optic disc. This condition mimics retinal detachment because the membrane attached to the optic disc can be mistaken for the retina. Subhyaloid heme and optic disc oedema can also be interpreted as retinal detachment. ${ }^{7}$ No false-negative results were observed in this study, although macula-on retinal detachment can be interpreted as a false-negative result.

This observational study had several limitations, including small sample size, few cases of retinal detachment, and wide confidence intervals. A study with a large sample size will decrease the confidence intervals, thereby consolidating these results.

The findings indicate that non-ophthalmic ultrasound is a highly reliable diagnostic method for excluding retinal detachment and may have a role in diagnosing retinal detachment in eyes with opaque media.

\section{Acknowledgements}

This study was supported by a research support grant from Prapokklao Hospital, Chanthaburi, Thailand. The authors thank Dr Urai Poonawagul for statistical analysis and www.editage.com for editing the manuscript for English language.

\section{References}

1. Munk PL, Vellet AD, Levin M, Lin DT, Collyer RT. Sonography of the eye. AJR Am J Roentgenol. 1991;157:1079-86.

2. Coleman DJ, Woods S, Rondeau MJ, Silverman RH. Ophthalmic ultrasonography. Radiol Clin North Am. 1992;30:1105-14.

3. Blumenkranz MS, Byrne SF. Standardized echography (ultrasonography) for the detection and characterization of retinal detachment. Ophthalmology. 1982;89:821-31.

4. Coleman DJ, Rondeau MJ, Silverman RH, Reinstein DZ, Lizzi F, Daly SW. Ultrasonography of the eye and orbit. 2nd ed. Philadelphia: Lippincott Williams \& Wilkins; 2005.

5. Blaivas M, Theodoro D, Sierzenski PR. A study of bedside ocular ultrasonography in the emergency department. Acad Emerg Med. 2002:9:791-9.

6. Blaivas M. Bedside emergency department ultrasonography in the evaluation of ocular pathology. Acad Emerg Med. 2000;7: 947-50.

7. Shinar Z, Chan L, Orlinsky M. Use of ocular ultrasound for the evaluation of retinal detachment. J Emerg Med. 2011;40:53-7.

8. Coleman DJ. Reliability of ocular tumor diagnosis with ultrasound. Trans Am Acad Ophthalmol Otolaryngol. 1973;77:OP677-86.

9. Silverman RH, Coleman DJ, Rondeau MJ, Woods SM, Lizzi FL. Measurement of ocular tumor volumes from serial, cross-sectional ultrasound scans. Retina. 1993;13:69-74.

10. Coleman DJ, Jack RL. B-scan ultrasonography of the retina and vitreous. Int Ophthalmol Clin. 1976;16:31-43.

11. Kurysheva NI, Maliuta GD, Erichev VP. Ultrasonography of lens crystalline in patients with primary open-angle glaucoma. Vestn Oftalmol. 1997;113:10-3. 